\title{
Francophonie, Médecine et Afrique
}

\section{Une réalité à préserver !}

\section{Pr. Francis Klotz}

(C) Springer-Verlag France 2011

Les XIV ${ }^{\text {es }}$ Journées d'hépato-gastroentérologie d'Afrique francophone viennent de se terminer à Brazzaville. Ce fut un succès ! Le palais des congrès de la capitale de la République du Congo a accueilli pendant trois jours 500 médecins et infirmiers venant de tous les pays francophones du continent africain ainsi que de France et de Belgique. Les échanges ont été nombreux et de qualité. La bonne tenue scientifique des conférences et communications, la qualité des ateliers furent au rendez-vous lors de cette réunion rendue agréable par l'accueil de nos amis congolais et la convivialité générale de notre Association Africaine Francophone de Formation Continue en Hépato-Gastro-Entérologie (AAFFCHGE).

Cela tient à deux éléments fondamentaux cimentant l'ensemble : la connivence des gastroentérologues du Nord et du Sud et la Francophonie dans cet espace où plus de 200 millions d'êtres humains se réclament de cette langue.

Comme le disait si bien Léopold Sedar Senghor : « $L a$ francophonie, c'est cet humanisme intégral qui se tisse autour de la terre: cette symbiose des énergies dormantes de tous les continents, de toutes les races qui se réveillent à leur chaleur complémentaire ».

L'originalité de la langue française ne réside pas dans une position de véhicule universel, diplomatique, scientifique et commercial comme l'est la langue anglaise. C'est un « trait d'union » entre les peuples des cinq continents, devenue au cours du temps instrument d'une culture internationale, réalisant un espace de "libérophonie » comme le disait Edgar Faure.

C'est Onésime Reclus qui définit la francophonie en 1880 comme «l'ensemble des espaces où la langue française est parlée dans le quotidien ».

Les circonvolutions de l'histoire ont permis la construction de cette entité au cours du temps. Dès le XVII ${ }^{\mathrm{e}}$ siècle, l'espace francophone a commencé à s'esquisser. Le maillage de la toile s'est fait progressivement sur le continent américain, au Moyen Orient et en Extrême Orient, par contre en Afrique le tableau est différent. L'espace francophone africain est la conséquence de l'expansion coloniale d'abord française puis plus récemment belge, ainsi que des guerres européennes depuis le $\mathrm{XVII}^{\mathrm{e}}$ siècle. Le continent noir a été considéré comme un monde à découvrir et à évangéliser. Les français ont pris pied sur la terre d'Afrique en y créant des comptoirs puis, progressivement, ont élargi leur zone d'influence vers l'intérieur des terres. Saint Louis du Sénégal a été créé dès 1641 , puis l'Ile de la Réunion appelée, Ile Bourbon, a été investie en 1649, l'Ile Maurice en 1715 ainsi que les comptoirs de la côte malgache.

La deuxième vague expansionniste coloniale s'est produite au $\mathrm{XIX}^{\mathrm{e}}$ siècle. Gallieni s'est rendu maître de Madagascar en 1897. Les grandes expéditions coloniales permirent à Bugeaud de vaincre Abdel Kader en Algérie, à Savorgan de Brazza de pénétrer profondément en Afrique centrale par le Congo, à Lamy de s'emparer de l'empire du Tchad, ce qui conduisit à la création de l'Afrique Occidentale Française (AOF) en 1895 et de l'Afrique Equatoriale Française en 1910. En 1914, l'empire colonial belge comprenait le Congo Belge puis après 1918, deux anciennes colonies allemandes : le Rwanda et le Burundi.

Ce n'est qu'après la Seconde Guerre mondiale que l'espace francophone commença à prendre toute sa signification avec l'individualisation d'une culture francophone universelle dont les chantres n'étaient plus français de métropole mais se nommaient : Léopold Sedar Senghor, Aimé Césaire, Habib Bourguiba.

Ainsi le français, tout d'abord instrument d'évangélisation puis de colonisation allait devenir, dans la deuxième moitié du $\mathrm{XX}^{\mathrm{e}}$ siècle, un instrument de libération puis de solidarité entre les peuples : "Ce merveilleux outil trouvé dans les décombres du régime colonial ! (L.S. Senghor).

Apanage d'une élite sous l'empire colonial, le français acquit une noble puissance après les indépendances en tant que langue officielle véhicule politique, de la diplomatie, de la science et de la culture. C'est la coexistence du français avec les langues autochtones qui détermine la francophonie, car il devient le trait d'union entre les différents pays et est ainsi générateur de force politique, économique et culturelle.

Le fait francophone réside donc dans la remarquable tolérance entre une langue d'apport et la langue maternelle ou nationale qui, pour l'africain, est l'élément privilégié de la manière d'appréhender le monde où il vit et son histoire. Il 
faut rappeler que même en France, le français n'a jamais été en position de langue unique mais côtoie la diversité des langues régionales.

Les soubresauts socio-politiques de plusieurs pays ont parfois mis à mal cette langue, la supprimant de l'enseignement scolaire ou l'interdisant au profit du russe comme à Madagascar. Toutefois ces péripéties, si elles laissent des stigmates de paupérisation linguistique, ne sont pas durables.

La Francophonie est soutenue par une ossature d'Institutions et d'Opérateurs que sont :

- L'Organisation internationale de la Francophonie (OIF), (anciennement Agence de Coopération Culturelle et Technique $[\mathrm{ACCT}])$;

- L'Assemblée parlementaire de la Francophonie (APF), qui est l'Assemblée consultative de la Francophonie (anciennement Association des Parlementaires de la Langue Française [AIPLF]) ;

- et les Opérateurs directs reconnus du Sommet, qui concourent, dans les domaines de leurs compétences, aux objectifs de la Francophonie :

- L'Agence universitaire de la Francophonie (anciennement Association des Universités Partiellement ou Entièrement de Langue Française [AUPELF]),

- TV5, la télévision internationale francophone,

- L'Université Senghor d'Alexandrie,

- L'Association internationale des maires responsables des capitales et des métropoles partiellement ou entièrement francophones (AIMF).

Ce grand trait d'union, devenu solide et incontournable, parfois malmené mais toujours vigoureux, est l'expression d'une culture internationale. Langue peu parlée par les peuples du continent africain où le pourcentage d'analphabétisme reste important, le français est une langue d'invention, de développement et de coopération scientifique et technique. Ce lien privilégié est une chance pour les pays qui en bénéficient. Il est parfois boudé ou galvaudé, perdant momentanément du terrain au profit de l'anglais.

Certains scientifiques et en particulier des médecins qui assurent qu'il est impossible de publier des travaux en français au risque d'être marginalisés, participent à la capitulation et vont dans le sens de « la normalisation anglo- saxonne ». Jusqu'à ce jour, la majorité des universitaires de l'espace francophone ont une formation, une culture et un raisonnement francophone, ce qui n'est aucunement antinomique avec les échanges positifs avec le monde anglophone, mais l'assimilation ou la destruction de l'un par l'autre serait un appauvrissement dommageable.

"L'union fait la force » et la différence avec l'adverse, mais il ne faut pas que l'ennemi soit davantage à l'intérieur par manque d'ouverture, conformisme ou frilosité.

La tentation d'unification est grande et les jeunes intellectuels africains sont attirés par les sirènes d'Outre Atlantique où leur identité est souvent diluée.

La francophonie en Afrique n'est pas servie par la paupérisation persistante d'un certain nombre de régions souffrant d'instabilité sociopolitique et de misère économique. Ces éléments mettent à mal l'enseignement, désorganisent les structures culturelles et font disparaître l'usage du français dans les tranches d'âge les plus jeunes qui devraient constituer le levain du progrès. Cette entité reste cependant bien vivante en tant qu'espace incontournable ayant un outil d'invention et de développement sans équivalent, mais la vigilance s'impose car la perte de puissance et de raison d'être de la langue française pourrait faire «perdre son âme » à l'espace francophone et précipiter ainsi nombre de régions fragiles dans des difficultés encore plus grandes.

Nos collègues africains francophones ont besoin de soutien et d'amitié : soutien scientifique et technique mais aussi compréhension du rôle qu'ils ont dans le développement de leur pays, eux qui sont les maillons transculturels entre leur société traditionnelle et la société occidentale scientifique et technique où ils ont progressé à nos côtés avant de retrouver les réalités de leur continent.

Coopérer, assister mais non se substituer dans un espace francophone de développement et de fraternité, tels doivent être les maîtres mots de «l'Association Africaine Francophone de Formation Continue en HépatoGastro-Entérologie » dont l'organe de diffusion scientifique «le Journal Africain d'Hépato-Gastroentérologie (JAHG)» est dynamique et performant.

Cette originalité doit rester notre fil conducteur. 